\title{
ALTERNATIVE REGULAR RINGS WITHOUT NILPOTENT ELEMENTS
}

\author{
MALCOLM F. SMILEY
}

Introduction. Interest in N. Jacobson's result that (associative) rings $R$ which satisfy

$$
a^{n(a)}=a
$$

( $n(a)$ an integer greater than one)

for every $a \in R$ are commutative ${ }^{1}[4$, p. 702] led Forsythe and McCoy to show that every (associative) regular ring without nilpotent ${ }^{2}$ elements is a subdirect sum of division rings [3]. The principal tool they used was a very general theorem of G. Birkhoff on subdirect unions in universal algebra [1]. In this note we shall prove the one additional fact which is needed to extend their argument to the alternative case. Our interest is not, of course, directed to the commutativity of alternative rings which satisfy (1), since this is an immediate consequence of Jacobson's result and the Theorem of Artin. In fact, such rings are associative. This consequence is well known if the additive order of no element of the ring is divisible by three. Our main result enables us to settle this exceptional case also. A corollary of our principal theorem is that every alternative algebraic algebra which has no nilpotent elements is the subdirect sum of alternative division algebras (cf. [4]). Whether this fact will find its place in a general theory of the structure of alternative algebraic algebras remains to be seen.

1. Preliminaries. Max Zorn calls a ring $R$ alternative in case, for every $a, b, c \in R$, the associator $(a, b, c)=a(b c)-(a b) c$ changes sign on interchange of two of its arguments $[7,8]$. Interest in these rings currently stems from a fundamental result of $\mathrm{R}$. Moufang in the foundations of projective geometry [5]. It is known to be sufficient to assume that $(a, a, b)=(b, a, a)=0$ [7]. Emil Artin has proved the following fundamental theorem.

THEOREM OF ARTIN. If $R$ is an alternative ring, then the subring of $R$ generated by two of its elements is an associative subring.

In this note we shall need to use the following identities

Presented to the Society, April 25, 1947; received by the editors February 20,1947, and, in revised form, March 7, 1947.

1 Numbers in brackets denote the references at the end of the paper.

${ }^{2}$ By a nilpotent element of a ring $R$ we mean a nonzero element $a \in R$ such that $a^{n}=0$ for a positive integer $n$. 


$$
\begin{aligned}
& (x(y z)) x=(x y)(z x), \\
& x(y(z y))=((x y) z) y
\end{aligned}
$$

which have been established for alternative rings by Zorn [8].

J. von Neumann [6] calls an (associative) ring $R$ regular in case for each $a \in R$ there is an $x \in R$ for which $a x a=a$. By the Theorem of Artin, this is a meaningful definition in alternative rings and we may speak henceforth of alternative regular rings.

2. The main result. In this section we shall prove the following theorem.

THEOREM. An alternative regular ring $R$ is a subdirect sum of alternative division rings if and only if $R$ has no nilpotent elements.

Proof. An examination of the argument of Forsythe and McCoy will show that associativity of three elements of $R$ which are not necessarily in a subring of $R$ generated by two elements of $R$ is used only in the proof of their Lemma 2 and in this case one of the three elements is idempotent. Our theorem is, then, a direct consequence of the Theorem of Artin, the arguments of Forsythe and McCoy, and the following lemma.

Lemma 1. If $e$ is an idempotent element of an alternative ring $R$ such that ex $=x e$ for every $x \in R$, then $e(x y)=(e x) y=x(y e)$ for ever $y x, y \in R$.

Before we prove this lemma we shall first prove the more general lemma.

LEMMA 2. If $x$ is an element of an alternative ring $R$ for which $x y=y x$ for every $y \in R$, then $x^{3} y=y x^{3}$ and $x^{3}(y z)=\left(x^{3} y\right) z=y\left(z x^{3}\right)$ for every $y$, $z \in R$.

Proof. That $x^{3} y=y x^{3}$ for every $y \in R$ is an immediate consequence of the Theorem of Artin. Now, using the identities (2.1) and (2.2), we may compute

$$
\begin{aligned}
(y z) x^{3} & =x^{3}(y z)=x[(x(y z)) x]=x[(x y)(z x)] \\
& =[(y x)(x z)] x=y\left(z x^{3}\right) .
\end{aligned}
$$

Thus $\left(y, z, x^{3}\right)=0$, and consequently all associators involving $x^{3}$ vanish.

Proof of Lemma 1. Take $x=e=x^{3}$ in Lemma 2.

Remark. Lemma 2 is related to a result of R. H. Bruck [2, p. 302] which states that the mapping $x \rightarrow x^{3}$ is an endomorphism of a commutative Moufang loop into its center. In our original version we 
noted that Bruck's result is valid for alternative rings in connection with our proof that alternative rings which satisfy (1) are associative. Our thanks are due to the referee who formulated Lemma 2, which, as we shall see, is useful in both situations.

3. The associativity of certain alternative rings. As we noted in the introduction, our main result is not needed to show that alternative rings which satisfy (1) are commutative. It is well known that a commutative alternative ring $R$ is associative if $3 a=0$ implies that $a=0$, for every $a \in R$. In this section we shall apply our main result to show that every alternative ring which satisfies (1) is associative. We first note that such a ring is regular and without nilpotent elements. By our theorem, such a ring is, then, a subdirect sum of commutative alternative division rings each of which satisfies (1). Now, an alternative division ring which satisfies (1) necessarily has finite prime characteristic (see [4, p. 702]). To prove the associativity desired it is sufficient to prove the following lemma.

LEMMA 3. If $R$ is a commutative alternative division ring whose characteristic is three and which satisfies (1), then $R$ is associative.

Proof. Let $a \in R$ and consider the subfield generated by $a$. This subfield is certainly finite since it is contained in the field

$$
K=\left[c_{1}+c_{2} a+\cdots+c_{n-2} a^{n-2} ; c_{1}, \cdots, c_{n-2} \in P_{3}\right],
$$

where $n=n(a)$ and $P_{3}=[0,1,2]$ is the prime field with three elements. Consequently, the field generated by $a$ is finite and $a^{q}=a, q=3^{k}$, with $k$ a positive integer. But then $a=b^{3}, b=a^{r}$, with $r=3^{k-1}$ and $b \in R$. The ring $R$ is, therefore, associative by Lemma 2 .

Note added in proof. (Received May 12, 1947.) R. H. Bruck has asked us to include the following theorem of his in our note because of its importance in geometric applications. Every commutative alternative division ring $R$ is associative and hence a field. For, if $a, b, c \in R$, we may write $(a b) c=t(a(b c))$, from which $a^{3} b^{3} c^{3}=t^{3} a^{3} b^{3} c^{3}$ follows by Lemma 2. It is well known that $3(a, b, c)=3(t-1)(a(b c))=0$. If $(a, b, c)$ is nonzero, then $t^{3}=1$ and $3(t-1)=0$. But then $(t-1)^{3}$ $=t^{3}-3 t(t-1)-1=0, t=1$, a contradiction. This result, together with our theorem, provides another proof of the conclusion reached in $\$ 3$. Irving Kaplansky has given (in conversation) still other proofs. Bruck's result and our theorem also imply that every regular commutative alternative ring is associative, since a regular commutative alternative ring has no nilpotent elements $[1$, p. 767]. We can then also show that every subdirectly irreducible commutative alternative ring 
without nilpotent elements is a field (cf. [1, Lemma 2]), but we shall not do this here.

\section{REFERENCES}

1. G. Birkhoff, Subdirect unions in universal algebra, Bull. Amer. Math. Soc. vol. 50 (1944) pp. 764-768.

2. R. H. Bruck, Contributions to the theory of loops, Trans. Amer. Math. Soc. vol. 60 (1946) pp. 245-354.

3. Alexandra Forsythe and N. H. McCoy, On the commutativity of certain rings, Bull. Amer. Math. Soc. vol. 52 (1946) pp. 523-526.

4. N. Jacobson, Structure theory for algebraic algebras of bounded degree, Ann. of Math. (2) vol. 46 (1945) pp. 645-707.

5. R. Moufang, Die Schnittpunktsätze des projecktiven speziellen Fiinfecknetzes in ihrer Abhängigkeit voneinander (Das A-Netz), Math. Ann. vol. 106 (1932) pp. 755-795.

6. J. von Neumann, On regular rings, Proc. Nat. Acad. Sci. U. S. A. vol. 22 (1936) pp. 707-713.

7. M. Zorn, Theorie der Alternativen Ringe, Abh. Math. Sem. Hamburgischen Univ. vol. 9 (1933) pp. 395-402.

8. - Alternative rings and related questions I: Existence of the radical, Ann. of Math. (2) vol. 42 (1941) pp. 676-686.

NORTHWESTERN UNIVERSITY

\section{A NOTE ON RELATIVELY PRIME SEQUENCES}

\section{RICHARD BELLMAN}

In volume 2 of Pólya-Szegö, Aufgaben und Lehrsätze aus der Analysis, pp. 133 and 342, there occurs the following result (appearing also in Hardy-Wright, Theory of numbers, p. 14):

THEOREM 1. No two numbers of the form $2^{2^{n}}+1, n=1,2, \cdots$, have a common divisor greater than 1 .

The numbers $2^{2^{n}}+1, n=1,2, \cdots$, are the well known Fermat numbers, which may be generated by iteration of the quadratic polynomial $\phi(x)=(x-1)^{2}+1$, choosing $x$ equal to 3 . This follows easily by induction, since, putting $\phi_{1}(x)=\phi(x), \quad \phi_{n+1}(x)=\phi\left(\phi_{n}(x)\right)$, if $\phi_{n}(x)=2^{2^{n}}+1$, then $\phi_{n+1}(x)=2^{2^{n+1}}+1$.

The above observation leads to the following result of which Theorem 1 is a special case:

Theorem 2. Let $\phi(x)$ be a polynomial in $x$ with integral coefficients

Received by the editors October 10, 1946, and, in revised form, February 18, 1947. 